\title{
Waugh Syndrome in a Child: A Case Report
}

\section{Chukwubuike Kevin Emeka*}

Pediatric Surgery Unit, Department of Surgery, Enugu State University Teaching Hospital, Enugu, Nigeria.

*Corresponding Author: Chukwubuike Kevin Emeka, Department of Surgery, Enugu State University Teaching Hospital, Enugu, Nigeria. Received date: 03 August 2021; Accepted date: 19 August 2021; Published date: 23 August 2021

Citation: Chukwubuike KE (2021) Waugh Syndrome in a Child: A Case Report. J Med Case Rep Case Series 2(9): https://doi.org/10.38207/JMCRCS/2021/0208133

Copyright: (C) 2021 Chukwubuike Kevin Emeka. This is an open-access article distributed under the terms of the Creative Commons Attribution License, which permits unrestricted use, distribution, and reproduction in any medium, provided the original author and source are credited.

\begin{abstract}
The combination of intussusception with intestinal malrotation is called Waugh syndrome. Waugh syndrome is rarely reported. We report a case of Waugh syndrome that presented with transanal protrusion of intussusception. A 7-months old female infant presented to the children's emergency room with a history of vomiting, fever, and passage of red currant jelly stool. He was evaluated, resuscitated, and had an unsuccessful hydrostatic reduction. Following failed non-operative treatment, he had laparotomy where intussusception with mobile and unfixed large bowel was confirmed. Manual reduction of the intussusception and treatment for the malrotation was rendered to the patient. Recovery and the postoperative period were uneventful. Waugh syndrome may be more common than is currently reported due to increased use of non-operative treatment.
\end{abstract}

Keywords: Case report, intussusception, intestinal malrotation, recurrence, Waugh syndrome.

\section{Introduction}

The association of intussusception with intestinal malrotation is called Waugh syndrome. Despite the high incidence of intussusception as a common cause of intestinal obstruction in infants in pediatric surgery practice, Waugh syndrome is seldom reported [1]. Up to 2014, only 63 cases of Waugh syndrome have been published [1]. However, Brereton et al reported a high incidence of malrotation among children who were operated upon for intussusception [2]. Among 37 children with intussusception, Brereton et al found the abnormal location of the duodenojejunal flexure in 15 children accounting for $40 \%$. One study from South Africa found Waugh syndrome in 6 out of 12 children that had intussusception [3]. The study suggested that malrotation by its nature is associated with the mobile right colon which may be a prerequisite for intussusception in children with Waugh syndrome [3]. This finding was supported by the report of Inan et al who found unfixed cecum and mobile colon in 2 patients with Waugh syndrome [4]. In Brereton study, the disparity between the high incidence of Waugh syndrome and the rarity of published reports suggest that many cases are undiagnosed: This may be due to the fact that most cases of intussusception are reduced nonsurgically by air or saline enema and radiological features of malrotation are not discovered in these circumstances [2]. The rarity of Waugh syndrome earns it being reported. To the best of our knowledge, this is the first report of Waugh syndrome coming from Sub-Saharan Africa.

\section{Case report}

A 7-months old female infant presented to the children's emergency room with one-week history of vomiting, fever, and passage of red currant jelly stool. Vomiting was non-projectile and vomitus was initially non-bilious but later become bilious. Fever was gradual in onset, low grade, and intermittent. Initial care of the patient was in a peripheral hospital where he stayed for 4 days without getting better. The patient had similar symptoms 2 weeks earlier and was treated by hydrostatic reduction which was "successful". On examination, she was febrile to touch, not pale, not in any painful distress but was mildly dehydrated. The temperature was $37.6^{\circ} \mathrm{C}$, pulse rate was 112 beats per minute, respiratory rate was 32 cycles per minute and weight was 6.5 kilograms. The abdomen was full, moves with respiration, and a mass was palpable in the left iliac fossa. Rectal examination showed an intussusception prolapsing through the anal opening (Figure 1). A working diagnosis of intestinal obstruction secondary to intussusception (with transanal protrusion) was made. 


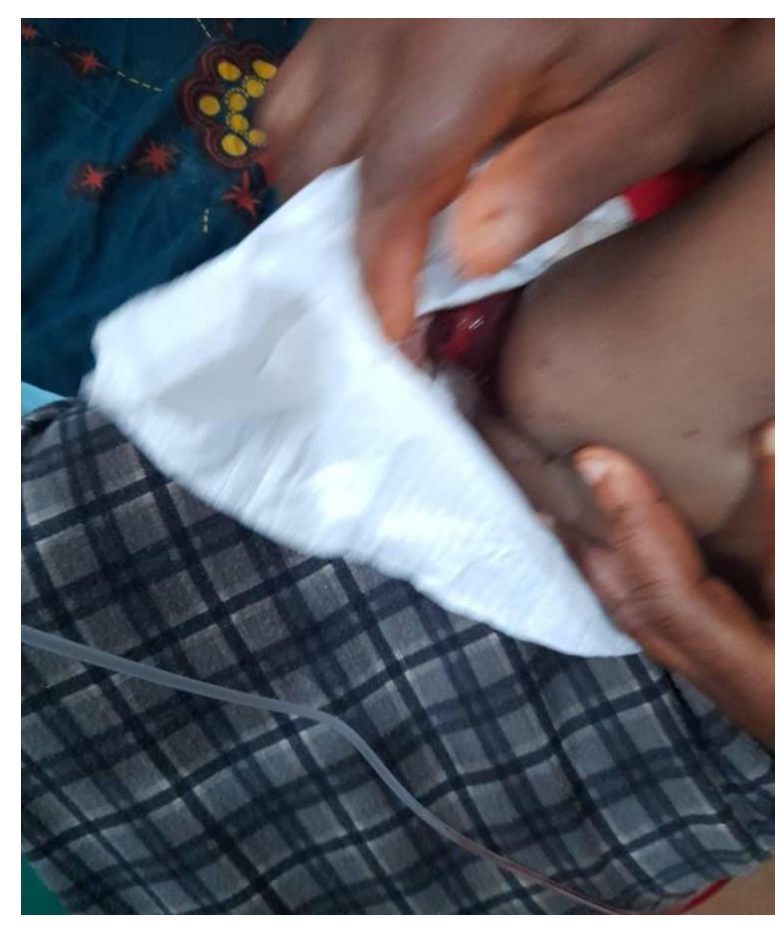

Figure 1: Transanal protrusion of intussusception

An abdominal ultrasound suggested the diagnosis of long segment intussusception. The hydrostatic reduction was unsuccessful. She was resuscitated, optimized, and taken to theatre for laparotomy. At laparotomy, the intussusception was confirmed and mobile unfixed caecum, ascending and descending colon noticed (figures $\mathbf{3}$ and $\mathbf{4}$ ).

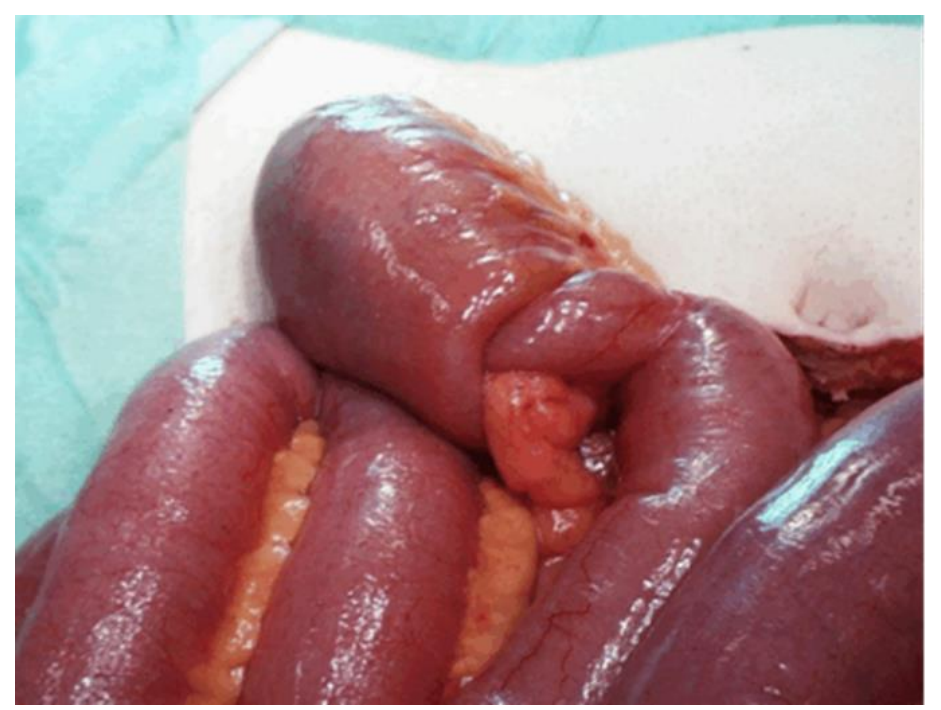

Figure 2: Intra-operative picture showing the intussusception

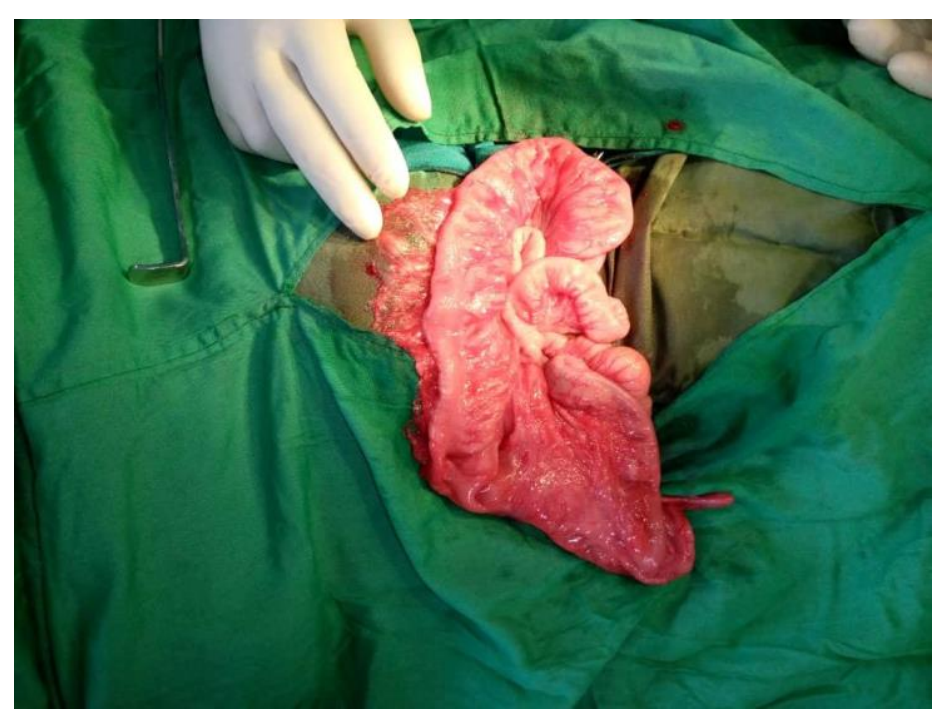

Figure 3: Intra-operative picture showing unfixed cecum, ascending, and descending colon.

The intussusception was manually reduced and the bowel was found to be viable. Release of congenital bands and widening of the mesentery was also performed and the abdomen was closed in layers.

\section{Discussion}

Intussusception may be associated with intestinal malrotation in a condition called Waugh's syndrome. Historically, in 1911, George E Waugh was the first to describe the association between intussusception and malrotation in a report of 3 boys with simultaneous intussusception and malrotation $[3,5]$.
The post-operative period was uneventful and the patient is currently being followed up in the clinic.

The age of our patient was 7 months. This is comparable to the report of other researchers [1]. Howbeit, Waugh syndrome can present at an age: It has been reported in a 28 -week neonate and in an 8-year-old child $[6,7]$. 
Laparotomy with manual reduction of the intussusception and Ladd procedure is the treatment of choice in Waugh syndrome [1]. This is meant to handle both pathologies (intussusception and malrotation) that are present in Waugh syndrome. The index patient was treated by laparotomy with manual reduction, the intestine straightened out, congenital bands divided, the small intestine placed on the right side of the abdomen and the colon placed on the left side. Appendectomy was not performed and the classical Ladd procedure was not followed. The futuristic need of the appendix may have informed this decision. However, the patient's parents were informed about the abnormal location of the vermiform appendix, in case of acute appendicitis in the future. It must be noted that there is a risk of recurrence of the intussusception in Waugh syndrome. Fixing the

\section{Conclusion}

Waugh syndrome may be more frequent than is currently reported. This may be due to the increasing practice of non-operative treatment of intussusception and failure to recognize this anomaly. Future research on intussusception should be based on preoperative imaging

\section{References}

1. Al-Momani H (2014) Waugh syndrome: a report of 7 patients and review of the published reports. Ann Saudi Med. 34(6): 527-531.

2. Brereton RJ, Taylor B, Hall CM (1986) Intussusception and Malrotation in infants: Waugh's syndrome. Br J Surg. 73(1): 5557.

3. Brecton VM, Hadley GP (2000) Waugh's syndrome: a report of six patients. Pediatr Surg Int. 16(5-6): 370-373.

4. Inan M, Basaran UN, Ayvaz S, Pul M (2004) Waugh's syndrome: report of two cases. J Pediatr Surg. 39(1): 110-101. bowel to the posterior abdomen is not advised because this may serve as a fulcrum for intestinal volvulus. Although the studied patient in the current report had laparotomy, it is noteworthy to state that a laparoscopic approach to Waugh syndrome has also been reported [8].

In our patients, there was a failed non-operative treatment. Other series on Waugh syndrome also reported a failure rate of $40 \%-100$ $\%$ of non-operative treatment $[3,6]$. The mobile unfixed cecum and large bowel may explain this high failure/recurrence rate of nonoperative treatment. Recurrence of intussusception following nonoperative treatment may be an indication of the presence of malrotation (Waugh syndrome).

on children with intussusception to determine the true incidence of Waugh syndrome. The exact location of the duodenojejunal junction on the upper gastrointestinal series defines the presence or absence of intestinal malrotation.

5. Waugh GE (1911) Referred penile pain in intussusception with notes of three cases. Lancet 1. 177(4579): 1492-1494.

6. Al-Jahdali A, Lees GM, Gay DP (2009) Colocolic intussusception in a preterm infant with intestinal malrotation. J Pediatr Surg. 44(12): e17-e18

7. Dawrant MJ, Lee JC, Ho CP (2005) Complex presentation of intussusception in childhood. Pediatr Surg Int. 21(9): 730-732

8. Hardy D, Howell C, Hatley R (2011) Laparoscopic approach to Waugh's Syndrome. Am Surg. 77(4): E78-79. 